\section{Interferência da tontura na qualidade de vida de pacientes com síndrome vestibular periférica}

\author{
Fernando F.Ganança', Ana Silvia O. Castro², \\ Fátima C. Branco ${ }^{3}$, Jamil Natour ${ }^{4}$
}

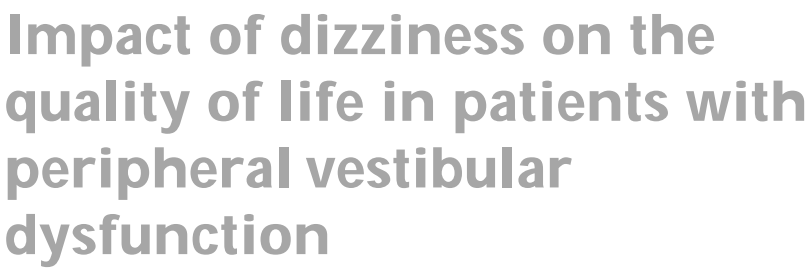

Resumo / Summary

\begin{abstract}
A
tontura é considerada um dos sintomas mais comuns entre adultos, podendo interferir na qualidade de vida dos pacientes que apresentam este sintoma. Objetivos: Descrever os resultados obtidos à aplicação do DHI brasileiro na população estudada e compará-los com a conclusão do exame vestibular dos respectivos pacientes. Formadeestudo: Clínico randomizado. Material e método: Vinte e cinco pacientes consecutivos que procuraram o Ambulatório da Disciplina de Otoneurologia da UNIFESP/EPM com queixa de tontura e hipótese diagnóstica de síndrome vestibular periférica submeteram-se ao questionário DHI brasileiro. Estes pacientes, adultos de ambos os sexos apresentaram idade variando entre 44 e 88 anos. 0 s resultados obtidos à aplicação do $\mathrm{DHI}$ foram comparados aos resultados do exame vestibular dos respectivos sujeitos. Resultados: Os pacientes avaliados apresentaram prejuízo na qualidade de vida devido à tontura, principalmente nos aspectos funcionais avaliados pelo DHI brasileiro. Os aspectos funcionais apresentaramse mais comprometidos nos pacientes com Síndrome Vestibular Periférica Deficitária (SVPD), independente do acometimento vestibular uni ou bilateral, e estas diferenças foram estatisticamente significantes. Conclusões: Os pacientes com tontura crônica apresentam prejuízo na qualidade de vida, em relação aos aspectos físicos, funcionais e emocionais verificados à aplicação do DHI brasileiro. Os pacientes com SVPD apresentam maior prejuízo na qualidade de vida nos aspectos funcionais à aplicação do DHI brasileiro, em relação aos pacientes com Síndrome Vestibular Periférica Irritativa.
\end{abstract}

Palavras-chave: tontura, vertigem, qualidade de vida, síndromes vestibulares.

Key words: dizziness, vertigo,

quality of life, vestibular diseases. izziness is one of the most common symptoms referred by adults. This symptom can decrease the dizzy patient's quality of life. Aim: To describe the results obtained from the application of the Brazilian version of the $\mathrm{DHI}$ and compare them with the conclusion of the vestibular test of the same patients. Study design: Clinical randomized. Material Method: Twenty five consecutive patients with chronic dizziness complaint were submitted to this questionnaire at the 0 toneurology Clinic of the Universidade Federal de São Paulo. These patients were female and male adults with ages ranging from 44 to 88 years. Results: All patients presented handicap in their quality of life due to dizziness, mainly in the functional aspects. The functional aspects were worse (differences were statistically significant) in patients with peripheral vestibular hypofunction syndrome than the patients with peripheral vestibular hyperfunction syndrome. Conclusions: 1 . Patients with chronic dizziness present abnormal quality of life because of dizziness, in relation to the physical, functional and emotional aspects, verified with the application of the Brazilian version of the DHI. Patients with peripheral vestibular hypofunction syndrome present worse quality of life because of dizziness related to the functional aspects than the patients with peripheral vestibular hyperfunction syndrome, verified at the application of the Brazilian version of the DHI.

\footnotetext{
${ }^{1}$ Professor Afiliado da Disciplina de Otoneurologia da UNIFESP/EPM. Professor do Programa de Pós-Graduação em Ciências da Reabilitação Neuro-Motora da UNIBAN. ${ }^{2}$ Fonoaudióloga Mestre em Ciências pelo Programa de Pós-Graduação em Ciências da Reabilitação Neuro-Motora da UNIBAN.

${ }^{3}$ Fonoaudióloga Doutoranda pelo Curso de Pós-Graduação em Neurociências e Comportamento pelo Instituto de Psicologia - da USP. Professora do Curso de Graduação em Fonoaudiologia da UNIBAN. ${ }^{4}$ Professor Afiliado da Disciplina de Reumatologia da UNIFESP/EPM.

Instituição: Universidade Federal de São Paulo / Escola Paulista de Medicina e Universidade Bandeirante de São Paulo Endereço para Correspondência: Dr. Fernando Ganança - Avenida Iraí, 438 cj. 113 Moema São Paulo SP $04082-001$ Telefax (0xx11)5044-6110/ 5532-1679

Artigo recebido em 14 de agosto de 2003. Artigo aceito em 11 de dezembro de 2003.
} 


\section{INTRODUÇÃO}

A tontura é considerada por alguns autores como um dos sintomas mais comuns entre adultos, principalmente em idosos. ${ }^{1-3}$

A tontura é a ilusão de movimento do próprio indivíduo ou do ambiente que o circunda. Este sintoma pode ser causado por uma disfunção em qualquer segmento dos sistemas relacionados ao equilíbrio corporal. Q uando a tontura adquire caráter rotatório é denominada vertigem. 0 dano ao sistema vestibular é a mais comum das causas orgânicas da vertigem. ${ }^{4,5}$

Além da vertigem, outros tipos de tontura ou instabilidade, juntamente com sintomas autonômicos secundários, tais como, sudorese, náusea e vômitos podem advir da disfunção do sistema vestibular ${ }^{6}$.

0 paciente com tontura habitualmente relata dificuldade de concentração mental, perda de memória e fadiga. A insegurança física gerada pela tontura e pelo desequilíbrio pode conduzir à insegurança psíquica, irritabilidade, perda de autoconfiança, ansiedade, depressão ou pânico ${ }^{7}$.

Muitos pacientes com tontura, deliberadamente restringem as atividades físicas, viagens e reuniões sociais, com 0 intuito de reduzir o risco de aparecimento destes sintomas desagradáveis e assustadores, e para evitar o embaraço social e o estigma que eles podem causar ${ }^{8}$.

Uma série de detalhes relatados pelos pacientes a partir da experiência de sentir tontura fornece indicações preliminares de como atitudes e crenças adotadas frente a um quadro vestibular podem limitar física e socialmente 0 indivíduo.

As conseqüências somato-psíquicas da vertigem recorrente, causada por desordens vestibulares, podem incluir ansiedade e ataques de pânico ${ }^{9}$, medo de sair sozinho ${ }^{10}$, interferência nas atividades diárias ${ }^{11}$ e sentimentos de estar fora da realidade e despersonalização ${ }^{12}$.

Os aspectos negativos descritos pelo paciente com tontura, associados à limitação e ao desconforto, causam conflito e embaraço social ${ }^{11}$ e também medo da incapacidade física ou de uma doença séria.

Pessoas com histórico psiquiátrico normal podem desenvolver sinais de ansiedade, pânico e agorafobia depois de uma crise vestibular. 9,10

Pode ser difícil diferenciar a contribuição das desordens físicas e psicológicas para as queixas de tontura, devido à considerável justaposição entre os sintomas de vertigem, pânico e ansiedade ${ }^{6}$.

É evidente que os fatores psicológicos são significantes para manter ou dar o valor à limitação e estresse causados pela vertigem.

O s testes do sistema vestibular não são particularmente sensíveis ou específicos para demonstrar a interferência psicológica no quadro clínico e no sofrimento do paciente com tontura. ${ }^{13}$
Segundo Hallam et al. (1988) ${ }^{14}$, Hallam, Hinchcliffe $(1991)^{15}$, é raro haver sincronismo entre o mal-estar causado pela vertigem e os resultados dos testes que fazem parte da avaliação otoneurológica.

0 exame otoneurológico é realizado para investigar funcionalmente o sistema vestibular, possibilitar a exclusão de alterações vestibulares relacionadas ao sistema nervoso central (SNC), além de confirmar os dados da história do paciente. 0 médico quer saber essencialmente sobre a condição das orelhas do paciente, da sua audição, da habilidade para responder aos testes de equilíbrio, sobre a presença ou ausência de nistagmo, e ter a certeza de que o funcionamento do SNC está satisfatório. Porém, algumas vezes o diagnóstico dos pacientes que sofrem de vertigem é feito somente por meio da história que este paciente traz. ${ }^{16}$

A possibilidade de se dispor de um instrumento capaz de estabelecer um perfil detalhado do paciente vertiginoso sobre o quanto sua qualidade de vida (QV) encontrase alterada, levou muitos pesquisadores a elaborar questionários e a testá-los, para tentar criar parâmetros de avaliação da QV. Além disso, definir quais os aspectos mais afetados nestes indivíduos pode auxiliar na escolha da terapia mais adequada.

A valiar a QV do paciente com a sua rotina alterada, devido à presença de sintomas vestibulares pode contribuir para estabelecer o tratamento mais adequado. Atualmente, a preocupação com o impacto que a doença ou a intervenção do profissional da saúde causa na QV do indivíduo é muito grande. ${ }^{17}$

Como a QV é um termo valorativo, inúmeras são as tentativas de mensurá-la e diversos instrumentos têm sido desenvolvidos, traduzidos e validados com este intuito. 0 termo QV aparece como descrito no MEDLINE desde 1977 e uma pesquisa nos bancos de dados traz cerca de 1000 instrumentos, apontando mais de 11.000 referências nos últimos 20 anos. A OMS possui um grupo voltado exclusivamente para esse fim, o World Health Organization - Quality of Life Group (WHOQ oL). ${ }^{18}$

$O$ conceito de saúde atualmente inclui aspectos funcionais, espirituais, cognitivos, emocionais e sociais. A OMS define a QV como "a percepção do in divíduo de sua posição na vida, no contexto de sua cultura esistema de valores nos quais ele está inserido e em relação aos seus objetivos, expectativas, padrões e preocupações". ${ }^{18}$

Existem dois tipos de questionários que podem ser utilizados para a avaliação de Q V: os questionários específicos para determinadas doenças ou partes do corpo, que são os preferidos pelos clínicos, e os genéricos, que avaliam a saúde geral do indivíduo. ${ }^{17}$

Em 1990, Jacobson e Newman ${ }^{19}$ elaboraram e validaram um questionário específico para tontura, o Dizziness Handicap Inventory (DHI), com o objetivo de avaliar a autopercepção dos efeitos incapacitantes impostos pela tontura. Estes autores afirmaram que o DHI requer pouco tem- 
po para ser aplicado e os resultados obtidos mostraram-se de fácil análise e interpretação, trazendo informações úteis para o planejamento e a realização do tratamento da tontura.

Jacobson, Newman (1990) ${ }^{19}$ consideraram que a quantificação dos efeitos dos tratamentos medicamentosos, cirúrgicos ou de reabilitação era muito difícil. Afirmaram que os testes convencionais para o diagnóstico são inadequados para avaliar os efeitos incapacitantes impostos pelas doenças do Sistema Vestibular.

Investigar a QV de modo sistematizado por meio de questionário traduzido e adaptado à língua utilizada em cada país é de fundamental importância. A adaptação cultural deste instrumento é fundamental para a utilização na população de um determinado país, que tem sua língua, seus hábitos e costumes. ${ }^{20}$

O DHI é o único questionário traduzido para a aplicação na população brasileira, até a presente data, com o objetivo de avaliar os prejuízos da QV em pacientes com tontura, denominado DHI brasileiro. Castro (2003) ${ }^{21}$; Ganança et al. (2003a) ${ }^{22}$ realizaram a adaptação cultural do questionário que constou de tradução do idioma inglês para o português e adaptação lingüística, revisão da equivalência gramatical e idiomática, adaptação cultural e reprodutibilidade intra e inter pesquisadores.

O s efeitos do tratamento das alterações do sistema vestibular, seja ele medicamentoso, cirúrgico ou de reabilitação, também podem ser acompanhados e mensurados por meio de questionários de QV.

Muitos estudos mostraram a importância de se avaliar os prejuízos da QV em pacientes vertiginosos com o intuito de quantificar os efeitos impostos pela vertigem nas funções de vida diária, além de auxiliar na escolha do tratamento e avaliação do mesmo. . $^{6,17,17,19,23-29}$

O s objetivos desta pesquisa são descrever os resultados obtidos à aplicação do DHI brasileiro e compará-los com a conclusão do exame vestibular dos respectivos pacientes.

\section{MATERIAL E MÉTODO}

Participaram da pesquisa vinte e cinco pacientes consecutivos que procuraram o ambulatório da Disciplina de O toneurologia do Departamento de O torrinolaringologia e Distúrbios da Comunicação Humana da Universidade Federal de São Paulo / Escola Paulista de Medicina, com queixa de tontura crônica (duração maior que três meses) e hipótese diagnóstica de síndrome vestibular periférica. Esta pesquisa foi aprovada pelo comitê de ética da UNIBAN.

Os pacientes adultos, do sexo masculino e feminino apresentaram idade variando entre 44 e 88 anos. Todos concordaram em participar da pesquisa, tendo assinado um termo de consentimento.

Este grupo de pacientes respondeu ao questionário DHI brasileiro, 21,22 adaptado culturalmente para a língua portuguesa utilizada no Brasil.
Este questionário avalia a interferência da tontura na qualidade de vida dos pacientes e é composto por vinte e cinco questões (Figura 1). As questões 01, 04, 08, 11, 13, 17 e 25 avaliam o aspecto físico, as questões $02,09,10,15,18$, $20,21,22$ e 23 avaliam 0 aspecto emocional e as questões $03,05,06,07,12,14,16,19$ e 24 avaliam 0 aspecto funcional.

O s resultados obtidos foram tabulados e, desta forma, obteve-se o perfil dos aspectos mais afetados para cada paciente: físico, emocional ou funcional.

As respostas dadas pelos pacientes receberam a seguinte pontuação: as respostas "sim" receberam quatro pontos, as respostas "não" não foram pontuadas (zero ponto), e as respostas "às vezes" receberam dois pontos.

0 escore total, bem como, os escores específicos de cada aspecto (físico, funcional e emocional) foram computados. Desta forma, o maior escore total obtido corresponde a cem pontos, situação em que se observa um prejuízo máximo causado pela tontura; e o menor, zero ponto, que revela nenhum prejuízo devido à tontura, na vida do paciente. Da mesma forma, avaliando-se cada aspecto individualmente, quanto maior o escore, maior o prejuízo causado pela tontura.

O s resultados obtidos com a aplicação do DHI brasileiro foram comparados com os resultados do exame vestibular para cada um dos vinte e cinco pacientes. Todos os pacientes já haviam sido submetidos ao exame vestibular de acordo com os procedimentos adotados pelo Ambulatório da Disciplina de Otoneurologia da UNIFESP-EPM.

O exame vestibular foi realizado no Setor de Vestibulometria, por meio do vectonistagmógrafo da marca BERGER, modelo VN 36, fabricado no Brasil, de acordo com os critérios de Mangabeira Albernaz et al. (1986) ${ }^{30}$, tanto na seqüência de realização, como nos parâmetros de interpretação.

Este exame constou das seguintes etapas: calibração biológica dos movimentos oculares, pesquisa do nistagmo espontâneo, pesquisa do nistagmo semi-espontâneo, pesquisa do rastreio pendular, pesquisa do nistagmo optocinético, pesquisa do nistagmo per-rotatório e pesquisa do nistagmo pós-calórico.

Os possíveis resultados encontrados são normal, síndrome vestibular periférica deficitária (SVPD), síndrome vestibular periférica irritativa (SVPI), síndrome vestibular central (SVC) ou síndrome vestibular mista (SVM). Neste exame também é possível detectar os lados acometidos, direito (D), esquerdo (E) ou ambos.

0 tratamento estatístico utilizado para avaliar possíveis associações entre síndrome vestibular periférica deficitária ou irritativa com os resultados do escore total, bem como o escore dos aspectos físico, funcional ou emocional, foi realizado por meio do teste do Qui-Q uadrado $\left(\aleph^{2}\right)$ para tabelas de associação, obedecendo-se às restrições de Cochnan e quando estas estavam presentes, utilizou-se 0 Teste Exato de Fisher. 


\begin{tabular}{|c|c|}
\hline 01. FI - Olhar para cima piora o seu problema? & $\operatorname{sim} \square$ não $\square$ às vezes $\square$ \\
\hline 02. EM - Você se sente frustrado(a) devido ao seu problema? & sim $\square$ não $\square$ às vezes $\square$ \\
\hline 03. FU - Voce̊ restringe suas viagens de trabalho ou lazer por causa do problema? & sim $\square$ não $\square$ às vezes $\square$ \\
\hline 04. FI - Andar pelo corredor de um supermercado piora o seu problema? & sim $\square$ não $\square$ às vezes $\square$ \\
\hline 05. FU - Devido ao seu problema, você tem dificuldade ao deitar-se ou levantar-se da cama? & sim $\square$ não $\square$ às vezes $\square$ \\
\hline $\begin{array}{l}\text { 06. FU - Seu problema restringe significativamente sua participação em atividades sociais tais } \\
\text { como: sair para jantar, ir ao cinema, dançar ou ir a festas? }\end{array}$ & $\operatorname{sim} \square$ não $\square$ às vezes $\square$ \\
\hline 07. FU - Devido ao seu problema, você tem dificuldade para ler? & sim $\square$ nåo $\square$ às vezes $\square$ \\
\hline $\begin{array}{l}\text { 08. FI - Seu problema piora quando você realiza atividades mais dificeis como esportes, dançar, } \\
\text { trabalhar em atividades domésticas, tais como varrer e guardar a louça? }\end{array}$ & sim $\square$ não $\square$ às vezes $\square$ \\
\hline $\begin{array}{l}\text { 09. EM - Devido ao seu problema, você tem medo de sair de casa sem ter alguém que o } \\
\text { acompanhe? }\end{array}$ & sim $\square$ não $\square$ às vezes $\square$ \\
\hline 10. EM - Devido ao seu problema, você se sente envergonhado na presença de outras pessoas? & sim $\square$ não $\square$ às vezes $\square$ \\
\hline 11. $\mathrm{Fl}$ - Movimentos rápidos da sua cabeça pioram o seu problema? & sim $\square$ não $\square$ às vezes $\square$ \\
\hline 12. FU - Devido ao seu problema, você evita lugares altos? & sim $\square$ não $\square$ às vezes $\square$ \\
\hline 13. FI - Virar-se na cama piora o seu problema? & $\operatorname{sim} \square$ não $\square$ às vezes $\square$ \\
\hline $\begin{array}{l}\text { 14. FU - Devido ao seu problema, é difícil para você realizar trabalhos domésticos pesados ou } \\
\text { cuidar do quintal? }\end{array}$ & sim $\square$ não $\square$ às vezes $\square$ \\
\hline $\begin{array}{l}\text { 15. EM - Por causa de seu problema, você teme que as pessoas achem que você está drogado(a) } \\
\text { ou bêbado(a)? }\end{array}$ & sim $\square$ não $\square$ às vezes $\square$ \\
\hline 16. FU - Devido ao seu problema é difícil para você sair para caminhar sem ajuda? & sim $\square$ não $\square$ às vezes $\square$ \\
\hline 17. FI - Caminhar na calçada piora o seu problema? & sim $\square$ não $\square$ às vezes $\square$ \\
\hline 18. EM - Devido ao seu problema, é difícil para você se concentrar? & sim $\square$ não $\square$ às vezes $\square$ \\
\hline 19. FU - Devido ao seu problema, é difícil para você andar pela casa no escuro? & sim $\square$ não $\square$ às vezes $\square$ \\
\hline 20. EM - Devido ao seu problema, você tem medo de ficar em casa sozinho(a)? & sim $\square$ não $\square$ às vezes $\square$ \\
\hline 21. EM - Devido ao seu problema, você se sente incapacitado? & sim $\square$ não $\square$ às vezes $\square$ \\
\hline 22. EM - Seu problema prejudica suas relações com membros de sua família ou amigos? & sim $\square$ não $\square$ às vezes $\square$ \\
\hline 23. EM - Devido ao seu problema, você está deprimido? & sim $\square$ não $\square$ às vezes $\square$ \\
\hline 24. FU - Seu problema interfere em seu trabalho ou responsabilidades em casa? & sim $\square$ não $\square$ às vezes $\square$ \\
\hline 25. FI - Inclinar-se piora o seu problema? & sim $\square$ não $\square$ às vezes $\square$ \\
\hline
\end{tabular}

\section{Legenda:}

$\mathrm{FI}=$ aspecto físicoFU $=$ aspecto funcional $\mathrm{EM}=$ aspecto emocional

Figura 1. DHI brasileiro (C astro, 2003; Ganança et al., 2003)

Nestas associações definiu-se para divisão dos escores o valor da mediana, pois evidentemente usando-se 0 Teste do Q ui-Quadrado ou o Exato de Fisher, que são testes não paramétricos, não seria válido usar a média aritmética.

0 nível de rejeição para a hipótese de nulidade foi fixado sempre em um valor menor ou igual do que 0,05 (5,00\%).

Quando a estatística calculada apresentou significância, usou-se um asterisco (*) para caracterizá-la. Caso contrário considerou-se não significante (N.S.)

\section{RESULTADOS}

O Grupo constituído por vinte e cinco pacientes que responderam ao $\mathrm{DHI}$ brasileiro apresentou idades que variaram entre 44 e 88 anos e média de idade de 66,88 anos. Dezenove indivíduos eram do sexo feminino e seis do masculino.

Todos os pacientes apresentaram prejuízo na qualidade de vida por causa da tontura, em pelo menos dois aspec- tos avaliados pelo $\mathrm{DHI}$ brasileiro. O s escores totais e dos aspectos físicos, funcionais e emocionais obtidos à aplicação do DHI brasileiro encontram-se descritos no Q uadro 1.

Os resultados do exame vestibular dos pacientes estão descritos no Q uadro 2. 0 exame vestibular esteve alterado em 19 dos 25 pacientes (76,0\%), sendo que 2 sujeitos apresentaram-se com SVPI à D, 4 com SVPI à E, 1 com SVPI bilateral, 8 com SVPD à $D, 2$ com SVPD à E e 2 com SVPD bilateral.

Os resultados alterados do exame vestibular dos pacientes, independente do lado afetado e do acometimento vestibular uni ou bilateral, foram analisados em relação à mediana do escore total e do escore dos aspectos físico, funcional e emocional, obtidos à aplicação do DHI brasileiro.

Não houve diferença estatisticamente significante em relação ao escore total, escore dos aspectos físico e emocional, obtidos na aplicação do DHI brasileiro, entre os pacientes com SVPD e SVPI. O escore do aspecto funcional foi maior entre os pacientes com SVPD ( $p=0,0399 *)$, (Tabela 1$)$. 
Quadro 1. Resultados obtidos quanto aos aspectos físicos, emocionais e funcionais e escore total, na aplicação do DHI brasileiro nos pacientes com tontura crônica

\begin{tabular}{|ccccc|}
\hline $\begin{array}{c}\text { identificação } \\
\text { do paciente }\end{array}$ & \multicolumn{4}{c}{ Escores dos aspectos físicos, funcionais e } \\
emocionais obtidos à aplicação do DHI brasileiro
\end{tabular}

Os pacientes com SVPD e SVPI com alteração unilateral ao exame vestibular foram comparados em relação ao escore total e os escores dos aspectos físico, funcional e emocional, obtidos à aplicação do DHI brasileiro.

Não houve diferença estatisticamente significante em relação ao escore total, escore dos aspectos físico e emocional, obtidos na aplicação do DHI brasileiro, entre os pacientes com SVPD e SVPI e alteração unilateral ao exame vestibular. 0 escore do aspecto funcional foi maior entre os pacientes com SVPD e alteração unilateral ao exame vestibular $(p=0,0262 *)$ (Tabela 2$)$

Os pacientes com SVPD e SVPI com alteração bilateral ao exame vestibular, foram comparados em relação ao escore total e os escores dos aspectos físico, funcional e emocional, obtidos à aplicação do DHI brasileiro.

Não houve diferença estatisticamente significante em relação ao escore total e escores dos aspectos físico, funcional e emocional, obtidos na aplicação do DHI brasileiro, entre
Quadro 2. Resultados obtidos ao exame vestibular dos pacientes com tontura crônica

\begin{tabular}{|cc|}
\hline $\begin{array}{c}\text { identificação } \\
\text { do paciente }\end{array}$ & resultado do exame vestibular \\
\hline 1 & SVPD à D \\
2 & Normal \\
3 & SVPD à E \\
4 & SVPD à E \\
5 & SVPD à D \\
6 & Normal \\
7 & Normal \\
8 & SVPD Bilateral \\
9 & SVPD à D \\
10 & SVPI à D \\
11 & SVPI Bilateral \\
12 & SVPD à D \\
13 & SVPD à D \\
14 & SVPI à E \\
15 & SVPI à D \\
16 & SVPI à E \\
17 & SVPI à E \\
18 & SVPD à D \\
19 & SVPD à D \\
20 & Normal \\
21 & SVPD Bilateral \\
22 & SVPI à E \\
23 & Normal \\
24 & Normal \\
25 & SVPD à D \\
à E = lado esquerdo & \\
& \\
Legenda:SVPD= síndrome vestibular periférica deficitária & \\
à DI = síndrome vestibular periférica irritativa \\
\\
\end{tabular}

os pacientes com SVPD e SVPI e alteração bilateral ao exame vestibular.

\section{DISCUSSÃO}

Os testes convencionais utilizados para o diagnóstico das alterações do sistema vestibular são insuficientes para avaliar todos os efeitos limitantes e/ ou incapacitantes, impostos pela tontura.

Desta forma, disponibilizar um instrumento para avaliar a qualidade de vida dos indivíduos que sofrem de vertigem e/ ou outras tonturas, é muito importante para os profissionais da saúde envolvidos com o tratamento destes pacientes.

O DHI foi desenvolvido em um ambiente cultural específico, os Estados Unidos da América, econômica, cultural e socialmente diferente de outros países, motivo pelo qual a sua utilização na população brasileira necessitou de 
Tabela 1. Distribuição dos pacientes com exame vestibular alterado, segundo o valor da mediana do escore do aspecto funcional obtido com a aplicação do DHI brasileiro

\begin{tabular}{cccc}
\hline $\begin{array}{c}\text { Aspecto } \\
\text { Funcional }\end{array}$ & $\begin{array}{c}\text { Síndrome Vestibular Periférica } \\
\text { Deficitária }\end{array}$ & Irnitativa & Total \\
\hline Mi $\leq 12$ & 4 & 6 & 10 \\
Mi $>12$ & 8 & 1 & 9 \\
\hline Total & 12 & 7 & 19 \\
\hline
\end{tabular}

Teste de Fisher

$p=0,0399$ *

Tabela 2. Distribuição dos pacientes com alteração unilateral ao exame vestibular segundo o valor da mediana do escore do aspecto funcional obtido com a aplicação do DHI brasileiro, na primeira entrevista da ENTREV1

\begin{tabular}{cccc}
\hline $\begin{array}{c}\text { Aspecto } \\
\text { Funcional }\end{array}$ & $\begin{array}{c}\text { Síndrome Vestibular Periférica } \\
\text { Deficitária }\end{array}$ & Irritativa & Total \\
\hline Mi d $\leq 12$ & 4 & 6 & 10 \\
Mi $>12$ & 6 & 0 & 6 \\
\hline Total & 10 & 6 & 16 \\
\hline
\end{tabular}

Teste de Fisher

$p=0,0262 *$

adaptação cultural. Estas diferenças não se resolvem apenas com uma simples tradução literal, sendo necessário levar em consideração o procedimento de adaptação cultural e a aplicação do questionário para que se determine a igualdade de características métricas entre a versão original e a traduzida.

0 questionário já traduzido e adaptado culturalmente à população brasileira foi aplicado em 25 pacientes com tontura crônica e hipótese diagnóstica de síndrome vestibular periférica, para avaliar a interferência da tontura na qualidade de vida destes pacientes.

A idade dos pacientes estudados variou entre $44 \mathrm{e}$ 88 anos. A média de idade foi de 66,88 anos, que é alta. Sabe-se que a tontura é mais prevalente nos indivíduos idosos, que tendem a apresentar um equilíbrio corporal mais comprometido em relação aos jovens. As alterações próprias do envelhecimento nos sistemas relacionados ao equilíbrio corporal, a maior possibilidade de doenças crônicodegenerativas e o uso crônico, por vezes múltiplo, de medicamentos, entre outros fatores, podem favorecer 0 aparecimento do sintoma tontura ou agravar a intensidade deste sintoma, provocando maior limitação física, funcional ou emocional nesta faixa etária. A maior prevalência de pacientes com idade mais avançada na população estudada pode ter influenciado na obtenção de maiores escores à aplicação do DHI brasileiro. Os pacientes foram incluídos de forma aleatória e fazem parte da população de pacientes que procura o Ambulatório de O toneurologia do Hospital São Paulo (UNIFESP/EPM).

Muitos dos pacientes desta pesquisa afirmaram em suas respostas ao DHI brasileiro limitação quanto às atividades sociais. Estas atividades referem-se aos aspectos funcionais avaliados pelo $\mathrm{DHI}$. Estes resultados são concordantes com as afirmações de Yardley, Putman (1992) ${ }^{8}$, que descreveram que muitos pacientes com tontura deliberadamente restringem as atividades físicas, viagens e reuniões sociais, com o intuito de reduzir o risco de aparecimento destes sintomas desagradáveis.

Os aspectos funcionais averiguados pelo $\mathrm{DHI}$ brasileiro investigaram a interferência da tontura em determinados movimentos dos olhos, da cabeça e do corpo, porém com enfoque na capacidade de desempenhar as atividades profissionais, domésticas, sociais, de lazer, e na independência ao se realizar determinadas tarefas como caminhar sem ajuda e andar pela casa no escuro.

Os aspectos emocionais avaliados pelo $\mathrm{DHI}$ brasileiro também se mostraram comprometidos pela tontura nos pacientes, indicando acometimento da estrutura mental dos pacientes que apresentam alterações do sistema vestibular. Este fato está de acordo com Pratt, Mackenzie (1958) ${ }^{9}$; Leavy, O'Leary (1947) ${ }^{10}$; Nobbs (1988) ${ }^{11}$ e Grisby, Johnston $(1989)^{12}$, que verificaram que os pacientes com alterações no sistema vestibular muitas vezes apresentam ansiedade associada a ataques de pânico, medo de sair sozinho e sentimentos de despersonalização, ressaltando a relação entre as alterações vestibulares e os aspectos emocionais.

Os aspectos emocionais do DHI brasileiro investigaram a possibilidade da tontura ter prejudicado a qualidade de vida dos pacientes gerando frustração, medo de sair desacompanhado ou ficar em casa sozinho, vergonha de suas manifestações clínicas, preocupação quanto à auto-imagem, distúrbio de concentração, sensação de incapacidade, alteração no relacionamento familiar ou social e depressão.

Os aspectos fisicos pesquisados pelo $\mathrm{DHI}$ brasileiro, apesar de serem avaliados por intermédio de um número menor de questões (7) em relação aos demais aspectos deste questionário, revelaram escores mais elevados.

Fielder et al. (1996) ${ }^{17}$ também verificaram que tanto para os homens quanto para as mulheres o desempenho das funções físicas foi significativamente mais afetado pelos efeitos da vertigem em relação aos outros aspectos avaliados pelo DHI. Jacobson, Calder $(2000)^{25}$ constataram que os pacientes com SVPD unilateral e SVPD bilateral também apresentaram maior pontuação (diferença estatisticamente significante) nos escores dos aspectos físicos em relação ao grupo de pacientes com ENG normal.

O s aspectos físicos do $\mathrm{DHI}$ brasileiro avaliaram a relação entre o aparecimento e/ou piora do sintoma tontura e os movimentos dos olhos, da cabeça e do corpo nos pacien- 
tes. 0 aparecimento de tontura em determinadas posições ou movimentos da cabeça é muito comum e pode ocorrer, por exemplo, em pacientes com vertigem posicional paroxística benigna, a mais comum das vestibulopatias. 0 utras vertigens posturais podem se manifestar com tontura à inclinação do corpo. Estímulos visuais (corredores de supermercado, obstáculos na calçada, movimentos de transeuntes) podem provocar ou agravar a tontura e também são pesquisados pelo $\mathrm{DHI}$ brasileiro.

O s escores encontrados à aplicação do DHI brasileiro foram comparados aos resultados alterados do exame vestibular, com o intuito de verificar a relação do prejuízo na qualidade de vida, devido à tontura, com os possíveis resultados obtidos à vestibulometria.

Nesta pesquisa, ao se avaliar os pacientes com alteração do exame vestibular, observou-se que houve associação estatisticamente significante entre a SVPD, independente do lado afetado e do comprometimento vestibular uni ou bilateral, e, também, a SVPD unilateral com os escores mais altos do aspecto funcional do DHI brasileiro.

O número reduzido de pacientes com diagnóstico de comprometimento bilateral da função vestibular, na população avaliada, não foi suficiente para que se pudesse demonstrar qualquer associação significante com os resultados obtidos à aplicação do DHI brasileiro.

Pode-se afirmar, portanto, que a associação significante entre SVPD, independente do lado afetado e do acometimento vestibular uni ou bilateral e os escores mais altos do aspecto funcional ao DHI brasileiro ocorreu devido ao número prevalente de casos com SVPD unilateral, que apresentaram tal comportamento.

Jacobson, Calder $(2000)^{25}$ também verificaram pior qualidade de vida nos pacientes com SVPD unilateral, relacionada, entretanto, ao escore total dos aspectos avaliados ao DHI. Encontraram ainda, pior qualidade de vida para os pacientes com SVPD bilateral em relação aos escores total e aos escores dos aspectos físicos pesquisados por este questionário.

As SVPD se correlacionam clinicamente com afecções vestibulares em que há diminuição total ou parcial da função vestibular, apresentando geralmente pior prognóstico em relação às SVPI. Distúrbios vestibulares como o schwannoma vestibular, a neurite vestibular, infecções e traumas da orelha interna, que cursam com destruição do epitélio neurossensorial do labirinto membranoso e/ ou das fibras dos nervos vestibulares, se manifestam caracteristicamente como SVPD e podem se apresentar com quadro clínico intenso e/ ou progressivo de tontura. Desta maneira, podem estar relacionados ao maior prejuízo na qualidade de vida, como observado nos pacientes desta pesquisa.

Jacobson Calder $(2000)^{25}$ verificaram correlação entre maior instabilidade postural apresentada nos subtestes de organização sensorial na plataforma da posturografia dinâmica computadorizada com os escores mais elevados no
DHI. O bservaram, também, que os pacientes com nistagmo espontâneo apresentaram maior prejuízo devido à tontura.

O DHI brasileiro pode ser aplicado na população brasileira, como um instrumento que permite a avaliação do prejuízo causado pela tontura na qualidade de vida dos pacientes vestibulopatas e, também, como método de acompanhamento da evolução clínica que verifique o efeito terapêutico obtido, seja devido a tratamento reabilitativo, medicamentoso, cirúrgico, etc.

Novos estudos devem ser realizados com o DHI brasileiro em pacientes com comprometimento vestibular bilateral para se verificar possível relação deste acometimento com a autopercepção da qualidade de vida destes pacientes.

\section{CONCLUSÕES}

Os pacientes com tontura crônica e hipótese diagnóstica de síndrome vestibular periférica ap resentam prejuízo na qualidade de vida, em relação aos aspectos físicos, funcionais e emocionais, avaliados à aplicação do DHI brasileiro. Os pacientes com SVPD apresentam maior prejuízo na qualidade de vida, nos aspectos funcionais avaliados pelo DHI brasileiro, em relação aos pacientes com SVPI.

\section{REFERÊNCIASBIBUOGRÁFICAS}

1. Kroenke K, Mangelsdorff AD. Common symptoms in ambulatory care: incidence, evaluation, therapy, and outcome. Am J Med 1989; 86:262-6.

2. Wodwell DA. Office visits to internists, 1989. Advance Data 1992; 209:1-10

3. Kroenke K, Lucas CA, Rosenberg ML et al. Causes of persistent dizziness: a prospective study of 100 patients in ambulatory care. Ann Intern Med 1992; 117:898-904.

4. Dix MR, Hood JD. Vertigo, Chichester: Wiley; 1984. 235p

5. Brandt T. Vertigo: Its Multisensory Syndromes. Londres: SpringerVerlag; 1990. 256p.

6. Yardley L, Masson E, Verschuur C, Haacket N, Luxon, L. Symptoms, Anxiety and Handicap in dizzy patients development of vertigo symptom scale. J Psychosom Res 1992; (36) 8:731-41.

7. Ganança MM, Caovilla HH. Desequilíbrio e reequilíbrio. In: Ganança MM.Vertigem tem cura? São Paulo: Lemos Editorial; 1998; p. 13-19.

8. Yardley L, Putman J. Quantitative analysis of factors contributing to handicap and distress in vertiginous patients: a questionnaire study. Clin Otolaryngol 1992; 17(3): 231-6.

9. Pratt RTC, McKenzie W. Anxiety states following vestibular disorders. Lancet 1958; 2:347-9.

10. Leavy I, O'Leary JL. Incidence of vertigo in neurologic conditions. Transactions of the American Otology Society 1947; 35:329-47.

11. Nobbs MB. Adjustment in Menière's Disease. In Kyle JG. Adjustment to Acquired Hearing Loss: Analysis, Change and Learning. Bristol: Centre for Deaf Studies; 1988.

12. Grisby JP, Johnston CL. Depersonalization, vertigo and Menière's disease. Psychol Rep 1989; 64:527-34.

13. Yardley L. Contribution of symptoms and beliefs to handicap in people with vertigo: A longitudinal study. $\mathrm{Br}$ J Clin Psychol 1994; 33:101-13.

14. Hallam RS, Beyts J, Jakes SC. Symptom reporting and objective test results: Explorations of desynchrony. In: Stephens SDG Stephens $\&$ Prasansuk S (eds). Advances in Audiology: measurement in hearing and balance. Karger: Basel, 1988. 327p. 
15. Hallan RS, Hinchcliffe R. Emotional stability: Its relationship to confidence in maintaining balance. J Psychosom Res 1991; 35:42130.

16. McNaboe E, Kerr A. Why history is the key in diagnosis of vertigo The pratictioner 2000; $244: 648-53$.

17. Fielder H, Denholm SW, Lyons RA, Fielder CP. Measurement of health status in patients with vertigo. Clin Otolaryngol 1996; 21:1246.

18. World Health Organization. International Classification of Impairment, Disabilities and Handicaps: a manual of classification relating to the consequences of disease. Geneva, World Health Organization; 1980.

19. Jacobson GP, Newman CW. The development of the Dizziness Handicap Inventory. Arch Otolaryngol Head Neck Surg 1990; 116:424-7.

20. Guillemin F, Bombardier C, Beaton D. Cross-cultural adaptation of health-related quality of life measures: literature review and proposed guidelines. J Clin Epidemiol 1993 Dec; 46(12):141732.

21. Castro ASO. Dizziness Handicap Inventory: adaptação cultural para o português brasileiro, aplicação e reprodutibilidade e comparação com os resultados à vestibulometria [tese]. São Paulo: Universidade Bandeirante de São Paulo; 2003.

22. Ganança FF, Castro ASO, Natour J, Branco FCA. Dizziness Handicap Inventory: cross-cultural adaptation to Brazilian Portuguese, its application, reproducibility and comparison with the vestibular evaluation results. Arch for Senso Neuro Sci Prac [periódico online] 2003a [cited $2003 \mathrm{Apr}$ 10]; (Apr 10):[6 screens]. Available from: URL: http: www.neurootology.org/search/?PHPSSESSID = (d599f3231e 4f0283564be73236f\&m $=c \& v=3$
23. Hazlett RL, Tusa RJ, Waranch HR. Development of an Inventory for Dizziness and Related Factors. J Behav Med 1996; 19(1): 7385

24. Heyning PHV, Wuyts FL, Claes J, Koekelkoren E, Van Laer C, Valke H. Definition, classification and reporting of Meniere's disease and its symptoms. Acta Otolaryngol (Stockh) 1997; 526:5-9.

25. Jacobson GP, Calder JH. Self-perceived balance disability/handicap in the presence of bilateral peripheral vestibular system impairment. J Am Acad Audiol 2000; 11(2): 76-83.

26. Silveira SR, Taguchi CK, Ganança FF. Análise comparativa de duas linhas de tratamento para pacientes portadores de disfunção vestibular periférica com idade superior a sessenta anos. Acta Awho [periódico online] 2002 [citado 2002 Fev 14]; (Junho 23): [14 telas]. Disponível em URL: http://www.acatawho.com.br/edicao/ conteúdo.asp?edi_id $=4 \& t p c$ id $=1 \&$ com_id $=$

27. Cavalli SS. Qualidade de vida em idosos com tontura que apresentam e não apresentam tontura [tese]. São Paulo: Universidade Bandeirante de São Paulo; 2003.

28. Ganança FF, Cavalli SS, Silva D, Serafini F, Perracini MR. Quality of life in elderly fallres with dizziness. Arch for Senso Neuro Sci Prac [periódico online] 2003b [cited 2003 Apr 10]; (Apr 10): [6 screens]. Avaliable from URL:http: //www. neurootology.

29. Cunha F. Interferência da tontura na qualidade de vida em pacientes com Doença de Ménière [tese]. São Paulo: Universidade Federal de São Paulo; 2003.

org/search/?PH PSESSI D $6 f \& m=c \& v=3$

30. Mangabeira Albernaz PL, Ganança MM, Caovilla HH, Ito YI, Novo NF, Juliano I. Aspectos Clínicos e Terapêuticos das Vertigens. Acta AWHO 1986;5(2):49-109. 